AIAA-2002-1744

\title{
Effect of Combined Loading and Fiber Type on the Strength of Polymer Matrix Composites
}

\author{
Chandra S. Yerramalli and Anthony M. Waas* \\ Composite Structures Laboratory, \\ Department of Aerospace Engineering, \\ University of Michigan, Ann Arbor, MI 48109-2140
}

February 12, 2002

\begin{abstract}
Glass and carbon fiber reinforced polymer matrix composite specimens of cylindrical shape were subjected to pure compression, pure torsion and combined compression-torsion loading. The specimens were of $50 \%$ fiber volume fraction, $V_{f}$, and the loading was done under displacement and rotational control in a proportional manner. The BudianskyFleck(BF) model for kinking of composites was specialized to the case of a solid cylinder. The BF model predicts a linear degradation in compressive strength under combined axial-torsional loading, which is in reasonable agreement with the experimental results obtained from carbon fiber composites. However, in the case of glass fiber composites, it was observed that the compressive strength did not degrade linearly as predicted by the BF model since kinking was not observed to be predominant. A new fracture mechanics based failure model to predict the behavior of glass fiber reinforced composites under combined compression-torsion loading has been developed. The new model predictions show a good correlation with the experimental data obtained from glass fiber composites. The present work shows the dependency of fiber type on the combined loading response of fiber reinforced polymer matrix composites.
\end{abstract}

\section{Introduction}

The compressive behavior of polymer matrix composite has been found to be very low as compared to its tensile strength. Thus, the compressive strength

\footnotetext{
* Graduate Research Assistant and Professor of Aerospace Engineering, respectively, University of Michigan. Assoc. Fellow AIAA, Copyright (C) 2002 by Anthony M. Waas. Published by the AIAA, with permission.
}

of composites has been the limiting factor in the design of composite structures. Based on the work of previous researchers [1-4] it is known that the uniaxial compression strength of polymer composites depends on the fiber type, matrix shear properties, fiber/matrix interface toughness and misalignments of the fibers. The effects of multiaxial loads on the compressive strength of composites has not been thoroughly investigated. The presence of shearing stresses, during the application of compressive loads on the specimen, induces additional misalignment in the fibers which would degrade the performance of the composite under compressive loads. Thus combined axial/torsional loading of solid cylindrical specimens will aid in understanding the effect of shear on the composite compressive strength. Previous work has been restricted to carbon composites at fixed volume fraction and under non-proportional remote loading. Considering the fact that glass composites have a distinctly different failure mechanism in compression as compared to carbon composites, the present work investigates both glass and carbon composites under combined axial torsional proportional loading. A fiber volume fraction of $50 \%$ was chosen since most commercially manufactured composites have a similar fiber volume fraction $\left(V_{f}\right)$.

\section{Experimental Details}

The composite specimens are solid cylinders of approximately $6.7 \mathrm{~mm}$ diameter and having a gage length of $12.7 \mathrm{~mm}$. Both the ends of the specimens were cut with a diamond saw to achieve a fine and smooth surface. The cylindrical specimens were made using E-glass (Vetrotex-certainteed) fibers of $24.1 \mu \mathrm{m}$ diameter and IM-7-12K carbon fibers(Hexcel corporation) of $5 \mu \mathrm{m}$ diameter with vinylester resin (Dow Derakane 411-C50) respectively. The specimens were cut with a fine dia- 
mond tip saw using a low speed cutting machine into lengths of $63.5 \mathrm{~mm}$. The gage length of the specimen was about $12.6 \mathrm{~mm}$ and the average diameter of the specimen was $6.7 \mathrm{~mm}$. The specimens were subjected to pure compression, pure torsion and combined compression-torsion under displacement control, rotation control and combined displacementrotational control loading respectively. The tests were performed on a Axial-Torsional MTS machine which had the capacity to simultaneously apply axial and torsional loads. The strains in the specimens were measured by attaching three strain gages on the specimen surface, two along the axial direction on opposite surfaces and the third one at an angle of $45^{\circ}$ to the vertical. The third strain gage was used to calculate the shearing strain and the two strain gages along the vertical were used to measure the axial strain in the specimen. Apart from the strain gage data, the cross head displacement, cross head rotation, axial force and the torque acting on the specimen were also measured. The combined compression torsion tests require that the specimen be gripped in such a manner that there is no slippage between the specimen surface and the interior of the grip while rotating the specimen and simultaneously applying an axial deformation.

\subsection{Testing Plan}

Initially pure compression and pure torsion tests were performed on the composite specimens, which correspond to the vertical and horizontal axis of the loading diagram as shown in Figure 1. As can be seen in Figure 1, different loading paths were adopted and the failure strength in each case was noted. The loading ratio was defined in terms of the axial displacement and the arc displacement as $\frac{\Delta}{R \theta}$, where $\Delta$ is the axial cross head displacement, $\theta$ is the applied cross head rotation and ' $R$ ' is the radius of specimen. This was repeated for both glass and carbon composites.

\section{Analytical Model}

\subsection{Kinking Model}

The conventional kinking model of BudianskyFleck(BF) modified to account for the remotely applied shear is based on the assumption that the shear stress varies linearly along the radius which is valid for a hollow thin walled specimen. In the present work solid cylindrical specimens were used for conducting the combined compression-torsion tests, consequently, an analytical model which accounts for a general distribution of shear stress along the radius of specimen was developed. For solid cylindrical specimens as shown in Figure 2, under pure torsion, Lyon [5] gave an expression for the shear stress $\tau$ in terms of the applied torque, $T$ and the rotation $\theta$ as shown below in equation(1).

$$
\tau_{R}=\frac{3}{2 \pi R^{3}}\left[T+\frac{\theta}{3} \frac{d T}{d \theta}\right]
$$

The expression for critical compressive stress in the presence of remotely applied shear stress, $\tau^{\infty}$ is derived as follows. As shown in Figure 3, the free body diagram of a small segment at a distance ' $r$ ' from the center of the cylindrical specimen is drawn in the deformed configuration. Then taking the moment equilibrium of the forces acting on the segment, the following expression for axial stress is obtained in terms of the remote shear stress, $\tau^{\infty}$, the shearing response of the composite material, $\tau(r)$, within an element of kinked fibers, the initial misalignment angle of the fibers, $\phi$, and the applied shear strain, $\gamma(r)$.

$$
\sigma_{z}=\frac{\tau(r)-\tau^{\infty}}{\phi+\gamma}
$$

In case of solid specimens, the shear stress $\tau(r)$ is not a linear function of ' $r$ ', hence its variation with ' $r$ ' is unknown and cannot be evaluated. However, the variation of shear stress, $\tau(r)$ is known as a function of shear strain, $\gamma$, which is a linear function of ' $\mathrm{r}$ ' given by $r \theta / l$, where $\theta$ is the rotation and $l$ is the specimen gage length. Therefore the first step in applying the BF model to the current problem is to express the shear stress as a function of shear strain, $\gamma$. This is achieved by expressing the specimen radius ' $r$ ' as $\frac{\gamma \theta}{l}$. Using the above substitution for ' $r$ ' and multiplying both sides of equation(2) by $r^{2} d r d \theta$ and integrating we obtain the following expression for critical compressive stress, $\sigma_{z}$, where, we substitute $T$ the applied torque for $\int_{0}^{R} \tau^{\infty} r^{2} d r d \theta$. Then,

$$
\sigma_{z}=\frac{\frac{1}{\gamma_{R}^{3}} \int_{0}^{\gamma_{R}} \tau(\gamma) \gamma^{2} d \gamma-\frac{T}{2 \pi R^{3}}}{\frac{\phi}{3}+\frac{\gamma_{R}}{4}}
$$

The integral term in equation(3) represents the shear response of the composite material and can be obtained from the pure torsion test data of the composite with similar fiber volume fraction. In equation(3) we substitute $\alpha \sigma_{z}$ for $\frac{T}{2 \pi R^{3}}$, where $\alpha$ is the loading ratio. Then we obtain an expression for $\sigma_{z}$ in terms of the shear response of the composite, loading ratio, $\alpha$, the misalignment angle, $\phi$, and the induced 
shear strain, $\gamma_{R}$ as given in equation(4). On solving equation(4) we get a limit load for $\sigma_{z}$ at some value of $\gamma$. This represents the critical kinking compressive stress for the composite.

$$
\sigma_{z}=\frac{\frac{1}{\gamma_{R}^{3}} \int_{0}^{\gamma_{R}} \tau(\gamma) \gamma^{2} d \gamma}{1+\frac{\alpha}{\frac{\phi}{3}+\frac{\gamma_{R}}{4}}}
$$

\subsection{Strain Energy Release Rate, $\mathcal{G}$}

In the following sections an energy based failure model has been developed for pure compression, pure torsion and combined compression-torsion loading of solid cylindrical composites. In essence, we have extended the Lee-Waas splitting model [2] for pure compression to the case of pure torsion and combined compression-torsion. For the sake of completeness, all three loading cases are discussed. However, complete derivations are presented only for the cases of pure torsion and combined compressiontorsion loading. Complete derivation for the case of pure axial compression loading can be referred to in the paper by Lee and Waas [2]. Consider a representative volume element(RVE) of the composite, consisting of a concentric cylinder of fiber and matrix, with a fiber radius, $r_{0}$ and an outer region of matrix with radius, $r_{1}$, and subjected to a external axial load, $P$ and torque, $T$, as shown in Figure 4. The outer radius of matrix is chosen to satisfy the fiber volume fraction requirement such that $r_{1}^{2}=V_{f} / r_{0}^{2}$. The outer matrix surface $\left(r=r_{1}\right)$ is assumed to be traction free. The height of the cylinder is taken to be ' $2 \mathrm{~L}$ ' with a interfacial crack of ' $2 \mathrm{l}$ ' embedded at the interface of fiber and matrix. The composite is assumed to have perfect bonding outside the crack region $(\mathrm{l} \leq \mathrm{z} \leq \mathrm{L})$ and $(-\mathrm{L} \leq \mathrm{z} \leq-\mathrm{l})$. The total potential energy is written as $\Pi=U-W$ where $U$ is the strain energy stored in the composite cylinder and $W$ is the work done by the external forces.

\subsubsection{Axial Loading}

The expression for strain energy release rate(SERR), $\mathcal{G}_{\text {Axial }}$ is derived below. The material is assumed to behave as a linear elastic material under the action of externally applied axial load, ' $P$ '. The strain energy release rate, $\mathcal{G}$ is defined as follows $\mathcal{G}=-\frac{d \Pi}{d A}$. The crack surface area, $A$ is taken to be $4 \pi r_{0} l$. Hence, the expression for strain energy release rate can be written as $\mathcal{G}=\frac{-1}{4 \pi r_{0}} \frac{d \Pi}{d l}$.

The total potential energy in case of

1. Displacement control (The applied displacement,
$\Delta$, is held fixed during crack propagation)

$$
\begin{gathered}
\Pi=U-W, U=\frac{1}{2} P \Delta, \text { and } W=0 \\
\mathcal{G}=-\frac{\Delta}{8 \pi r_{0}} \frac{d P}{d l}
\end{gathered}
$$

Using the definition of compliance, $C=\Delta / P$

$$
\begin{aligned}
\frac{d P}{d l} & =-\frac{P^{2}}{\Delta} \frac{d c}{d l} \\
\text { Hence, } \mathcal{G} & =\frac{P^{2}}{8 \pi r_{0}} \frac{d c}{d l}
\end{aligned}
$$

2. Load control (The applied load, $\mathrm{P}$, is held fixed during crack propagation)

$$
\begin{gathered}
\Pi=U-W, U=\frac{1}{2} P \Delta, \text { and } W=P \Delta \\
\mathcal{G}=\frac{P}{8 \pi r_{0}} \frac{d \Delta}{d l}
\end{gathered}
$$

Using the definition of compliance, $C=\Delta / P$

$$
\begin{array}{r}
\frac{d \Delta}{d l}=P \frac{d c}{d l} \\
\text { Hence, } \mathcal{G}=\frac{P^{2}}{8 \pi r_{0}} \frac{d c}{d l}
\end{array}
$$

Note that the expression for strain energy release rate, $\mathcal{G}$ is same under load control as well as displacement control due to the assumption of linear elastic material behavior.

The expression for the change in compliance with respect to crack length, $d c / d l$ of the composite is taken from Lee and Waas [2] and is as follows.

$$
\frac{d c}{d l}=\frac{2}{\pi r_{0}^{2}}\left(\frac{1}{\delta}-\beta\right)
$$

where,

$$
\begin{gathered}
\delta=E_{f}+E_{m}\left(\frac{1}{V_{f}}-1\right) \\
\alpha=\left[\frac{2\left(1+\nu_{f}\right)\left(1-2 \nu_{f}\right)}{E_{f}}\left(V_{f}^{-1}-1\right)+\frac{2\left(1+\nu_{m}\right)\left(1-2 \nu_{m}+V_{f}^{-1}\right)}{E_{m}}\right]^{-1} \\
\beta=\left[E_{f}+\left(V_{f}^{-1}-1\right)\left\{E_{m}+4 \alpha\left(\nu_{f}-\nu_{m}\right)^{2}\right\}\right]^{-1}
\end{gathered}
$$


In computing the above quantities, only the stress states of the cracked and uncracked regions are considered, whereas a region near the crack tip of finite size $\epsilon$ is not considered. In the present analysis, the above expression for $d c / d l$ is used, even though this expression for $d c / d l$ was obtained by neglecting the crack tip stress field. However, as explained later, for steady state condition, equation (7) is exact. In the region $\epsilon$ (Figure 5), the stress state is influenced by the crack tip field. However, under steady state condition for self similar crack growth, this region translates with the crack tip resulting in an increase of $l$ and a corresponding decrease of $(L-l)$. Thus, while the axial contraction and compliance given by $\Delta$ and $c$ above are approximate due to the negligence of the crack tip field, the rate of compliance change due to crack advancement, given by $d c / d l$ is exact, since the ' $\epsilon$ ' region is invariant with respect to crack length. This fact enables us to calculate $\mathcal{G}$ accurately for steady state crack propagation. When the crack is small, initially the compliance change with respect to crack length, $d c / d l$, is dependent on the size of $\epsilon$, but, as the crack length increases, $d c / d l$, becomes independent of crack length and attains the steady state value provided in equation (7). The derivation of $d c / d l$ including the dependence on $\epsilon$ is given in [6], for non-steady state crack growth.

From (7), the energy release rate per unit area is obtained as

$$
\mathcal{G}=\frac{P^{2}}{4 \pi^{2} r_{0}^{3}}\left(\frac{1}{\delta}-\beta\right)
$$

When $\mathcal{G}$, becomes equal to twice the critical interfacial surface energy $\left(\gamma_{f}\right)$ the initial crack propagates;

$$
\mathcal{G}=2 \gamma_{f}
$$

Thus,

$$
\sigma_{c r}=\sqrt{\frac{8 V_{f}^{2} \gamma_{f}}{r_{0}(1 / \delta-\beta)}}
$$

\subsubsection{Misaligned Fibers}

The expression for axial compliance for the case when the fiber is misaligned in the cracked region can be obtained by considering a slightly imperfect fiber with an initial imperfection of $w_{0}(x)$ and using kinematics that are appropriate for geometrically nonlinear beam theory as described by Lee and Waas [2]. Then the relation between axial contraction $\Delta$, and fiber load, $P_{f}$, can be obtained as,

$$
\frac{\Delta_{1}}{l}=\frac{-P_{f}}{A_{f} E_{f}}\left[1+\frac{A_{0}^{2} A_{f}}{2 I_{f}}\right],
$$

where, $A_{f}$ is the fiber cross-sectional area, $I_{f}$ is the area moment of inertia and $A_{0}$ is the imperfection magnitude. From this relation an effective axial stiffness for the fiber is obtained and is given below

$$
(E A)_{e f f}=\frac{E_{f} A_{f}}{1+\frac{A_{0}^{2} A_{f}}{2 I_{f}}},
$$

Clearly, when the imperfection vanishes, one recovers the perfect fiber axial stiffness $(E A)_{f}$. In the present work, the imperfection amplitude is chosen to correspond to an initial misalignment angle of $2^{0}$. Detailed derivation of the axial splitting model is presented in [2]

\subsubsection{Torsional Loading}

Strain energy release rate expressions for a cylindrical specimen of linear elastic and non-linear elastic material under the action of pure torsion, ' $T$ ' are developed below. Closed form expressions of strain energy release rate for a linear elastic material behavior are obtained in terms of the compliance of the material.

The strain energy release rate expression for a cylinder of radius $r_{0}$, under rotational (displacement control) and torsional(load control) loading is derived as follows.

1. Rotational control (The applied rotation, $\phi$, is held fixed during crack propagation)

$$
\begin{gathered}
\Pi=U-W, U=\frac{1}{2} T \phi, \text { and } W=0 \\
\mathcal{G}=\frac{\phi}{8 \pi r_{0}} \frac{d T}{d l}
\end{gathered}
$$

Using the definition of compliance, $C=r_{0} \phi / T$

$$
\mathcal{G}=\frac{T^{2}}{8 \pi r_{0}^{2}} \frac{d c}{d l}
$$

2. Torque control (The applied torque, $\mathrm{T}$, is held fixed during crack propagation)

$$
\Pi=U-W, U=\frac{1}{2} T \phi, \text { and } W=T \phi
$$




$$
\mathcal{G}=\frac{T}{8 \pi r_{0}} \frac{d \phi}{d l}
$$

Using the definition of compliance, $C=r_{0} \phi / T$

$$
\mathcal{G}=\frac{T^{2}}{8 \pi r_{0}^{2}} \frac{d c}{d l}
$$

The expression for $d c / d l$ for a solid circular cylinder of length ' $2 l$ ' and made of linear elastic material is $2 /(G J)$ where $G$ is the shear modulus of the material and $J$, is the polar moment of inertia given by $\pi r_{0}^{4} / 2$. Hence, we can write the expression for strain energy release rate as follows

$$
\mathcal{G}=\frac{T^{2}}{4 \pi r_{0}^{2} G J}
$$

When the strain energy release rate becomes equal to the critical fracture toughness, $2 \gamma_{f}$ the crack will propagate leading to failure. The critical failure stress in torsion can then be written in terms of $\gamma_{f}$ as follows.

$$
\tau_{c r}=4 \sqrt{\gamma_{f} G / r_{0}}
$$

For a non-linear elastic material, the expression for strain energy release rate, $\mathcal{G}$, under displacement control loading can be obtained as follows.

1. Displacement control ( The applied rotation, $\phi$, is held fixed during crack propagation)

$$
\begin{array}{rl}
\Pi= & U-W \\
U & =\int_{V} \int_{0}^{\gamma} \tau(\zeta) d \zeta d V=4 \pi l \int_{0}^{r_{1}} \int_{0}^{\gamma} \tau(\zeta) d \zeta r d r \\
W & 0
\end{array}
$$

In equation (13), the inner integral is a known function of $\zeta$, where $\zeta$ is a dummy variable for the purpose of integration. But, the outer integral in ' $r$ ' has to be first converted in terms of shearing strain $\gamma$ since for a solid cylinder we do not know the distribution of $\tau$ with respect to the radius of cylinder, $r$. Using the relations $\gamma=r \nu$, and $d r=$ $d \gamma / \nu$, in the integral of equation (13) we get the following integral. Here, ' $\nu$ ' is the twist per unit length given by the ratio $\phi / l$

$$
U(\gamma)=4 \pi l \int_{0}^{\gamma_{r_{1}}}\left(\int_{0}^{\gamma} \tau(\zeta) d \zeta\right) \gamma / \nu^{2} d \gamma
$$

Differentiating equation (15) with respect to ' $l$ ' we get

$$
\frac{d U}{d l}=4 \pi \int_{0}^{\gamma_{r_{1}}}\left(\int_{0}^{\gamma} \tau(\zeta) d \zeta\right) \gamma / \nu^{2} d \gamma
$$

where $\zeta$ is a dummy variable and $\gamma_{r_{1}}$ is shear strain at radius, $r_{1}$.

\subsubsection{Compression-Torsion loading}

Consider a concentric cylinder of fiber and matrix with a debond of length ' $2 l$ ' as shown in Figure 4 . We can divide this representative volume element(RVE) into a cracked region of linear elastic fiber and a non-linear elastic matrix and an uncracked region of non-linear elastic composite. Now let this RVE be subjected to a combined set of axial and torsional loads as shown in Figure 4. ' $P$ ' is the axial compressive load and ' $T$ ' is the torsional load. The total strain energy release rate under combined compression-torsion loading can be written as the sum of axial and torsional strain energy release rate contributions. It should be noted that we are implicitly assuming that the non-linear torsional response is unaffected by the presence of axial stress.Thus,

$$
\mathcal{G}_{\text {Total }}=\mathcal{G}_{\text {Axial }}+\mathcal{G}_{\text {Torsion }}
$$

In the present case, it was observed during the experiments that the axial stress - strain curve remains linear upto the point of failure even under combined compression-torsion loading. Hence, strain energy release rate, $\mathcal{G}_{\text {Axial }}$ is obtained based on linear elastic material behavior. In torsion the strain energy release rate, $\mathcal{G}_{\text {Torsion }}$, is obtained by adding the strain energy release rate contributions from a linear elastic fiber and non-linearly elastic matrix in the cracked region and a non-linearly elastic composite in the uncracked region under torsional load.

$$
\mathcal{G}_{\text {Torsion }}=-\frac{1}{4 \pi r_{0}} \frac{d U}{d l}
$$




$$
=-\frac{1}{4 \pi r_{0}}[\underbrace{\frac{d U}{d l} \text { fiber }_{\text {matrix }}+\frac{d U}{d l}}_{\text {crackedregion }}+\underbrace{\frac{d U}{d l_{\text {composite }}}}_{\text {uncrackedregion }}]
$$

Now, explicit relations for the terms entering in equation (18) are given below for both the cracked region and the uncracked region. In the cracked region the fiber is assumed to be linear elastic hence the term $d U / d l_{f i b e r}$ can be written as

$$
\frac{d U}{d l}_{\text {fiber }}=\frac{\tau^{2} \pi^{2} r_{0}^{2}}{2 G_{f i b e r}}
$$

Also, in the cracked region matrix is debonded from the fiber and is modeled as a non-linear elastic material for the calculation of $d U / d l_{\text {matrix }}$.

Matrix

$$
\frac{d U}{d l}_{\text {matrix }}=4 \pi \int_{\gamma_{r_{0}}}^{\gamma_{r_{1}}}\left(\int_{0}^{\gamma} \tau_{m}(\zeta) d \zeta\right) \gamma / \nu^{2} d \gamma
$$

Uncracked region

In the uncracked region away from the crack tip we model the composite as a homogenous nonlinear elastic material. Thus, the expression for $d U / d l_{\text {composite }}$ can be written as follows

\section{Composite}

$$
\frac{d U}{d l}_{\text {composite }}=4 \pi \int_{0}^{\gamma_{r_{1}}}\left(\int_{0}^{\gamma} \tau_{c}(\zeta) d \zeta\right) \gamma / \nu^{2} d \gamma
$$

From equations $(9,19-21)$ we can write an expression for the total strain energy release rate(SERR) of the composite as

$$
\begin{aligned}
\mathcal{G}_{\text {Total }}= & \frac{\sigma_{c}^{2} r_{0}}{4 V_{f}^{2}}\left(\frac{1}{\delta}-\beta\right)+\frac{\tau^{2} \pi^{2} r_{0}^{2}}{2 G_{\text {fiber }}} \\
& +\frac{1}{r_{0}} \int_{\gamma_{r_{0}}}^{\gamma_{r_{1}}}\left(\int_{0}^{\gamma} \tau_{m}(\zeta) d \zeta\right) \gamma / \nu^{2} d \gamma+ \\
& \frac{1}{r_{0}} \int_{0}^{\gamma_{r_{1}}}\left(\int_{0}^{\gamma} \tau_{c}(\zeta) d \zeta\right) \gamma / \nu^{2} d \gamma \quad(22
\end{aligned}
$$

When the total strain energy release rate, $\mathcal{G}_{\text {Total }}$ becomes equal to $2 \gamma_{f}$, then splitting failure occurs. Therefore, we can write the above equation in terms of the surface energy term $\gamma_{f}$ and the compressive strength of the composite, $\sigma_{c}$, under combined loading as,

$$
\begin{aligned}
\gamma_{f}= & \frac{\sigma_{c}^{2} r_{0}}{8 V_{f}^{2}}\left(\frac{1}{\delta}-\beta\right)+\frac{\tau^{2} \pi^{2} r_{0}^{2}}{4 G_{f i b e r}} \\
& +\frac{1}{2 r_{0}} \int_{\gamma_{r_{0}}}^{\gamma_{r_{1}}}\left(\int_{0}^{\gamma} \tau_{m}(\zeta) d \zeta\right) \gamma / \nu^{2} d \gamma+ \\
& \frac{1}{2 r_{0}} \int_{0}^{\gamma_{r_{1}}}\left(\int_{0}^{\gamma} \tau_{c}(\zeta) d \zeta\right) \gamma / \nu^{2} d \gamma
\end{aligned}
$$

\section{Solution Procedure}

Equation (23) relating the fracture toughness to the axial stress and shear stress acting on the composite is solved numerically to obtain the critical value of compressive stress and the corresponding value of shear stress. The input parameters required to solve equation (23) are the elastic and geometric properties of fiber, the shear response of pure matrix, the shear response of the composite and the fiber volume fraction of the composite. Table 1 shows the properties used in the present failure model. The shear response was incorporated in the analytical solution by using the Ramberg-Osgood fit parameters obtained from [7] and are given in Table 2. With the above input parameters, the equation is solved for the critical value of shear stress or compressive stress for different values of loading ratios, ' $\kappa$ ', where $\kappa$ is defined as $\sigma / \tau$. The critical value of shear stress or compressive stress is attained when for a particular value of $\kappa$, the right hand side of equation (22) exceeds the left hand side value of critical fracture toughness, $\gamma_{f}$. A range of $\gamma_{f}$ values of $0.1224 K J / \mathrm{m}^{2}$ to $0.0408 \mathrm{KJ} / \mathrm{m}^{2}$ has been used to study the effect of fracture toughness on the predicted failure envelope.

\begin{tabular}{cccc}
\hline & & & \\
& $E_{f}(\mathrm{MPa})$ & $\mu_{f}(\mathrm{MPa})$ & $r_{0}(\mathrm{~mm})$ \\
\hline & & & \\
Glass fiber & 72000 & 29508 & 0.012 \\
Vinylester & 3585 & 1318 & \\
\hline
\end{tabular}

Table 1: Properties of glass fiber and vinylester resin

\section{Discussion}

The results obtained from the combined axial compression-torsional loading of solid circular 


\begin{tabular}{ccccc}
\hline & $V_{f}$ & $\mu_{c}(\mathrm{MPa})$ & $\mathrm{A}$ & $\mathrm{n}$ \\
\hline & & & & \\
Glass composite & 0.5 & 3260 & 68.68 & 12.44 \\
Vinylester & 0 & 1318 & 65.44 & 7.9603 \\
\hline
\end{tabular}

Table 2: Ramberg-Osgood fit for shear stress-strain curve of glass/vinylester and pure vinylester specimens

cylindrical specimens of glass/vinylester and carbon/vinylester composites indicated that the remotely applied shear stress caused a degradation in the composite compressive behavior leading to a decrease in the failure strength. However, there was a difference in the response of carbon composites to remote shear stress as compared to that of glass composites. The carbon composites show a approximately linear reduction in compressive strength as the remote shear stress is increased, which matches with the prediction of the modified $\mathrm{BF}$ model, equation(4). This can be observed in Figure 6, where the axial compressive strength is plotted against a normalized torque defined as $\frac{T}{2 \pi R^{3}}$. The glass/vinylester composites were found to be initially unaffected by the remotely applied shear stress. After the remote shear stress reached a critical value a significant drop in compressive strength was observed as can be seen in Figure 7. The experimental data show that for high values of $\Delta / r \theta$, the compressive strength of specimens was found to increase. This could be attributed to the fact that for small rotations of cross sections, the applied shear strain acts in a manner to reduce the misalignments thus leading to an increase in compressive failure strength.

The results in Figure 7 indicate that the MBF kinking model predictions are inaccurate for the glass composites which were tested under combined compression-torsion loading. The glass composites tested are made of fibers of diameter $24.1 \mu \mathrm{m}$ and fail by splitting as observed in experiments instead of failing in a kinking mode. This observation indicates the need for a model that explicitly accounts for the effect of fiber diameter on the composite compressive strength. Such a model should also predict the mode of failure, based on the magnitude of compressive stress that is required ro induce the failure mode in question. The present fracture mechanics based model which is applicable to splitting failure is used to compare the predicted and the observed experimental values for the failure envelope under a combined state of compression-torsion loading. The value of fracture toughness as a function of fiber volume fraction of the composite is not available but a initial value of $\gamma_{f}=0.1224 \mathrm{KJ} / \mathrm{m}^{2}$ has been chosen to predict the failure envelope. The failure envelope predictions based on this value of $\gamma_{f}$ were found to be very high since this value of $\gamma_{f}$ corresponds to the fracture toughness of pure epoxy and is more suitable for lower volume fractions. Also, the failure of glass composites at high volume fractions was seen to be a combination of splitting and kinking. Hence, the failure model was used to predict the failure envelope for a range of fracture toughness $\left(\gamma_{f}=0.1224 K J / m^{2}, \gamma_{f}=0.0612 K J / m^{2}, \gamma_{f}=\right.$ $\left.0.0408 K J / \mathrm{m}^{2}\right)$. The correlation between the predicted stresses and the experimentally obtained data is good as indicated in Figure 8. The better correlation between the predicted value of compressive strength and experimentally observed strength for a lower value of $\gamma_{f}$ could indicate that the interfacial fracture toughness reduces at higher fiber volume fraction. The new model captures the trend of the failure data and the correlation is much better in the range where the loading ratio, $\kappa$ is high. At lower values of $\kappa$ the model predicts higher values of splitting compressive strength than the observed experimental values. However, it should be noted that the failure mode in case of higher values of shear stress(i.e. lower $\kappa$ ) was not pure splitting but a combination of matrix crushing and splitting, which is not taken into account by the current fracture model. To better understand the initial insensitivity of the glass composites to remote shear stress, a plot of axial strain energy release rate(SERR) and torsional SERR as a precentage of the total SERR with respect to the applied shear stress, $\tau$, is shown in Figure 9. It can be seen from Figure 9 that upto a significant value of remote shear stress, $\tau$, the axial SERR contribution to the total SERR is nearly $100 \%$ indicating that remotely applied shear stress does not play a role in inducing failure as seen in experiments. When the shear stress $\tau$ reaches a critical value, which is about $40-50 \mathrm{MPa}$ for the glass composites there is a sudden rise in the contribution from the torsional SERR and it reaches a peak value of $100 \%$ for pure torsion loading.

\section{Conclusions}

The present experimental work has revealed that the response mechanism of glass fiber composites to remote shear stress is different from that of the 
carbon fiber composites. The results indicate that the conventional Budiansky-Fleck model is applicable to carbon fiber composites, where the failure is due to buckling of fibers in an inelastic matrix. In glass composites there seems to be a critical value of remote shear stress beyond which the compressive strength of the composite degrades very rapidly. This difference in sensitivity to the remote shear stress motivated us to develop a new fracture mechanics based failure model which captures the initial insensitivity of compressive strength to shear stress and the subsequent steep drop in compressive strength when the remotely applied shear stress is very high. Apart from the difference in sensitivity to remote shear stress exhibited by carbon and glass composites, the failure mechanism is also found to be different. In case of the glass composite specimens tested it was found that the failure mechanism changed from splitting to kinking and a combination of matrix crushing and splitting was observed as the remote shear stress increased. Ideally a single model which can effectively tackle the mode transition from splitting to kinking failure and vice-versa would be the best model. Conceivably, such a model would have to be implemented numerically, perhaps using the finite element method.

\section{ACKNOWLEDGMENTS}

The authors are grateful to the Army Research Office for supporting this research. Dr. Bruce LaMattina is the ARO scientific monitor.

\section{$\underline{\text { References }}$}

[1] N. A. Fleck. Compressive failure of fiber composites. In Advances in applied mechanics, volume 33, pages 43-117. Academic Press, New York, 1997.

[2] S. H. Lee and A. M. Waas. Compressive response of fiber reinforced unidirectional composites. International Journal of Fracture, 100:275306, 1999.

[3] T. J. Vogler and S. Kyriakides. Inelastic behavior of an as4/peek composite under combined transverse compression and shear. part I: experiments. International Journal of Plasticity, 15:783-806, 1999.

[4] T. J. Vogler, S. Y. Hsu, and S. Kyriakides. Composite failure under combined compression and shear. International Journal of Solids and Structures, 37:1765-1791, 2000.

[5] R. E. Lyon. Shear strength of a ductile material from torsion test of solid cylinders. Journal of Testing and Evaluation, 19(3):240-243, 1991.

[6] Chandra S. Yerramalli and A. M. Waas. Compressive splitting failure of composites using modified shear lag theory. To appear in International Journal of Fracture, 2002.

[7] Chandra S. Yerramalli and A. M. Waas. In-situ matrix shear response using torsional test data of fiber reinforced unidirectional polymer composites. To appear in ASME Journal of Engineering Materials and Technology, 2002.

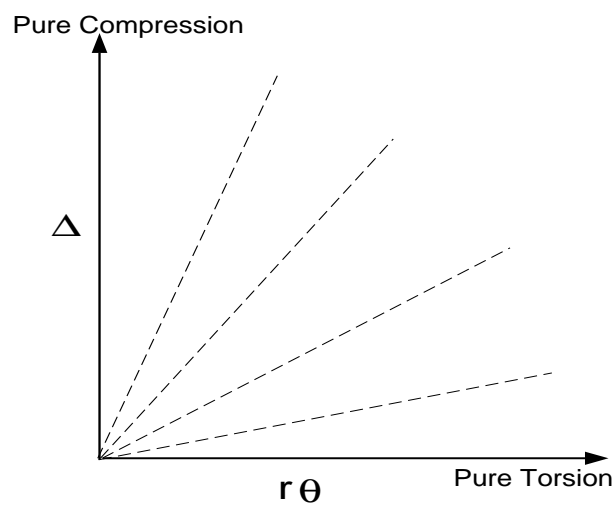

Figure 1: Different loading paths in a displacement/rotation control test

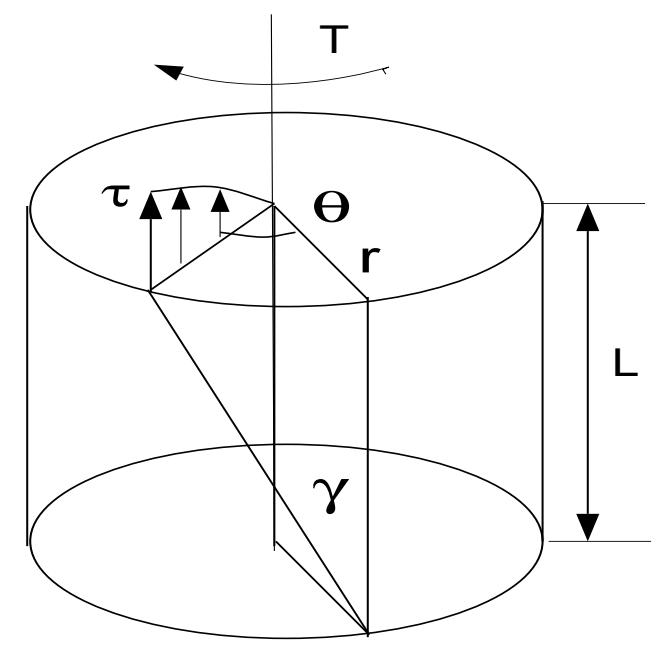

Figure 2: Pure torsion of a circular cylinder 


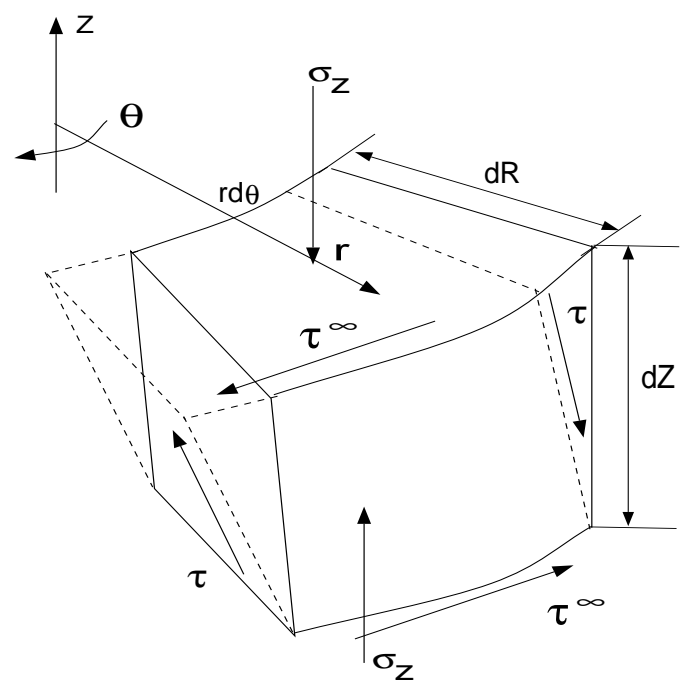

Figure 3: Free body diagram of a kinked segment of a cylinder under combined compression and torsion

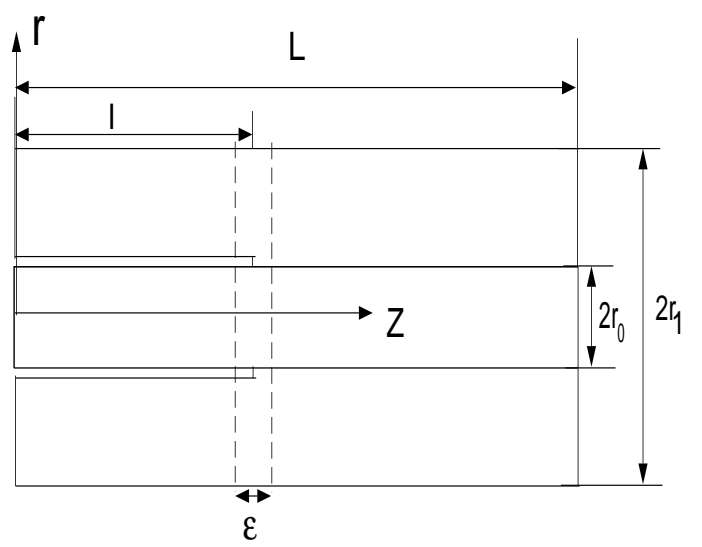

Details of the crack region

Figure 5: Schematic cross section of fiber-matrix cylinder showing the crack tip details

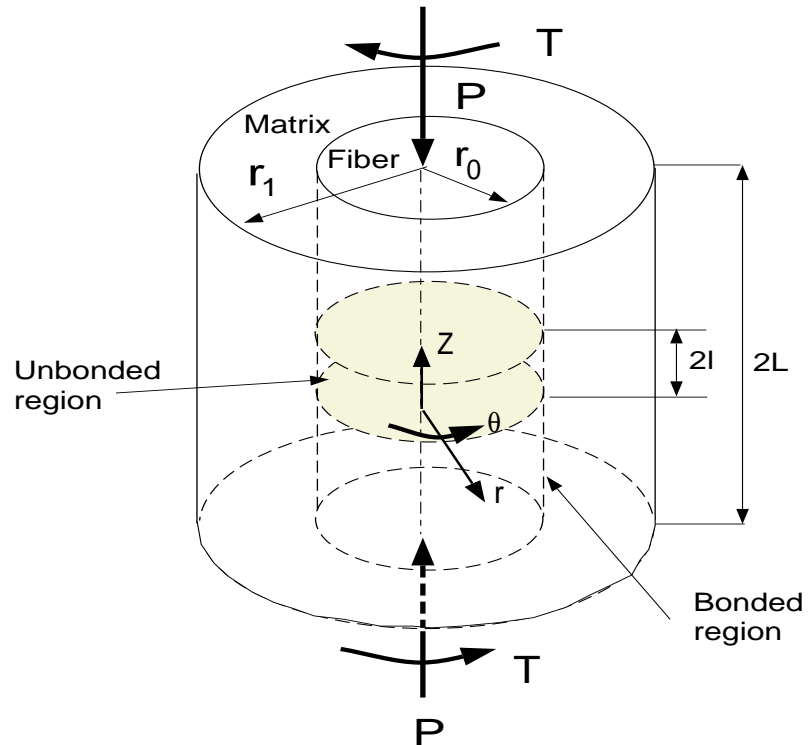

An RVE of composite with initial crack

Figure 4: Composite cylinder under compressiontorsion loading

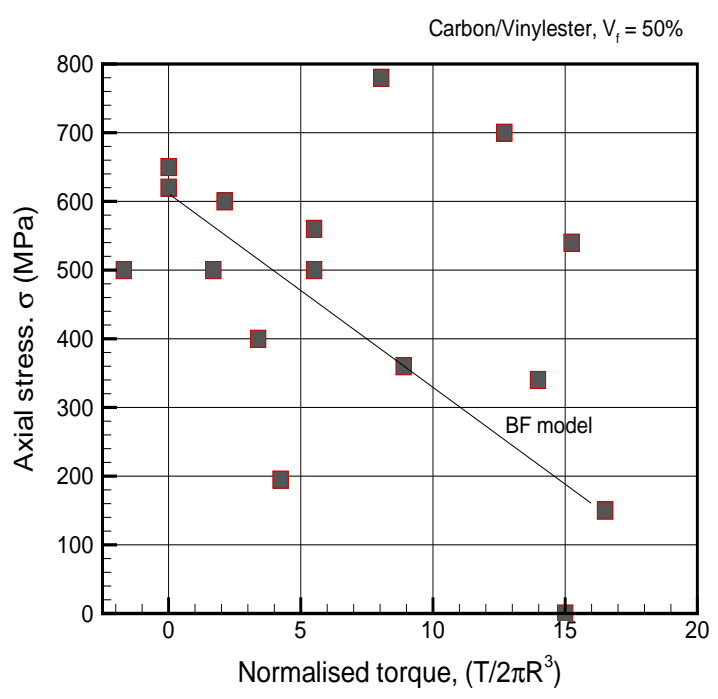

Figure 6: Combined compression-torsion failure plot for carbon/vinylester composite of $V_{f}=50 \%$ 


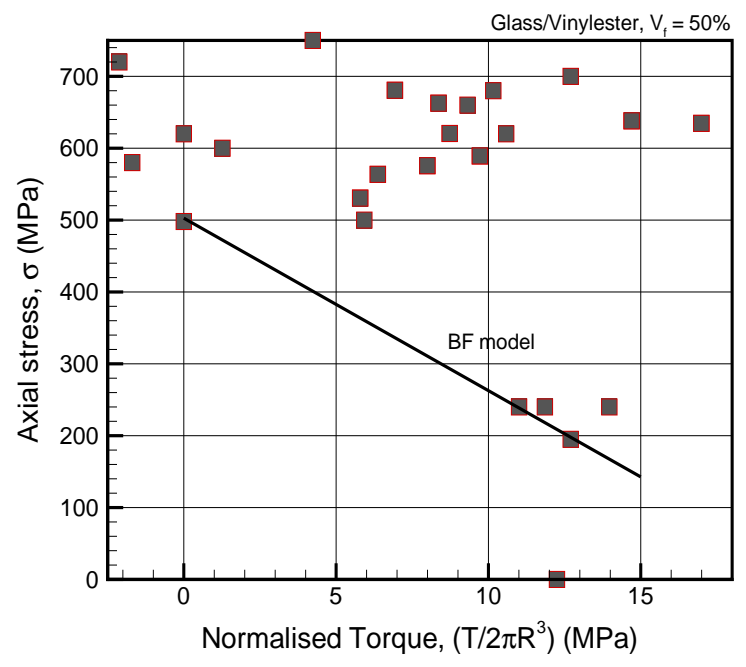

Figure 7: Combined compression-torsion failure plot for glass/vinylester composite of $V_{f}=50 \%$
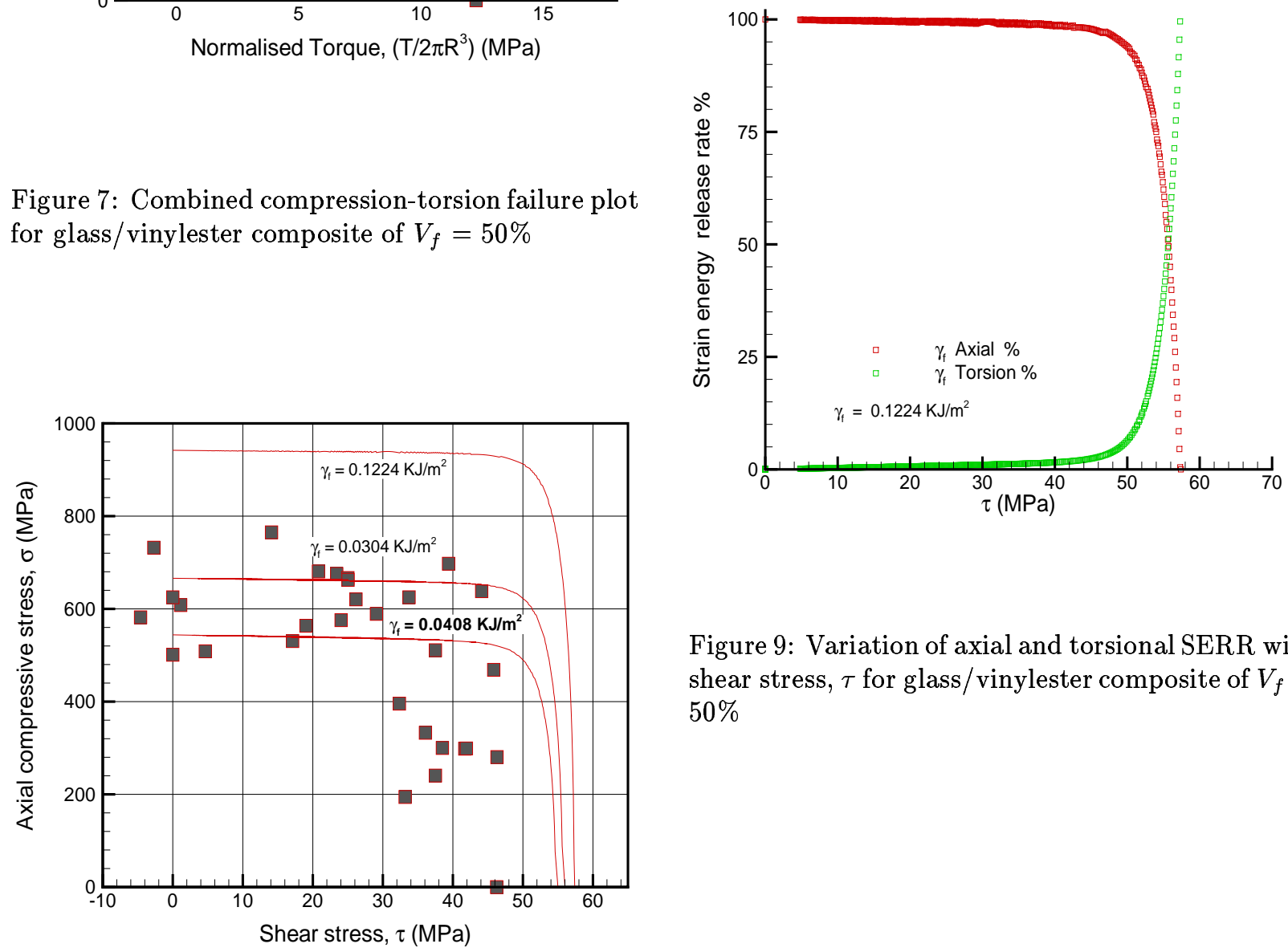

Figure 9: Variation of axial and torsional SERR with shear stress, $\tau$ for glass/vinylester composite of $V_{f}=$ $50 \%$

Figure 8: Comparison of the new fracture critieria with experimental data for a glass/vinylester composite of $V_{f}=50 \%$ 